Vol 11, Issue 1, 2018

\title{
ANTIFUNGAL ACTIVITY OF A SECONDARY METABOLITE OF AZADIRACHTA INDICA AND ITS DERIVATIVES - AN IN SILICO STUDY
}

\begin{abstract}
SIMHADRI VSDNA NAGESH ${ }^{1 *}$, MUNIAPPAN $\mathbf{M}^{2}$, KANNAN ${ }^{3}$, VISWANATHAN $\mathbf{S}^{4}$
${ }^{1}$ Department of Pharmacology, Bharath University, Tagore Medical College and Hospital, Chennai, Tamil Nadu, India. ${ }^{2}$ Department of Pharmacology, Sree Balaji Medical College and Hospital, Chennai, Tamil Nadu, India. ${ }^{3}$ Department of Microbiology, Tagore Medical College and Hospital, Chennai, Tamil Nadu, India. ${ }^{4}$ Department of Pharmacology, Meenakshi Medical, College and Hospital, Chennai, Tamil Nadu, India. Email: simhadrii117@gmail.com.
\end{abstract}

Received: 22 August 2017, Revised and Accepted: 07 October 2017

\section{ABSTRACT}

Objective: This study was aimed to inhibit the 1, 3 -glucan synthase with azadirachtin or with the derivatives by docking method.

Methods: The homology model of the protein 1, $3 \beta$ glucan synthase was prepared with "easy modellar" using query sequence and template and it was validated with procheck of Ramachandran plot. The ligand was selected from the PubChem database, and the .sdf file was downloaded which was converted to another file format with open babel. The .pdb files of protein and ligand were uploaded for rough docking with iGEMDOCK, and finally, the accurate docking was made with autodock vina. The docked poses were visualized with PYMOL then saved. The derivatives of the ligand were generated with SWISS ADME, free online software, and selected the derivative for docking.

Results: The results obtained from iGEMDOCK and Autodock Vina were tabulated. It was found out that the Azadirachtin and the derivatives are effective in binding $1,3 \beta$ Glucan synthase and thereby inhibiting the formation and integrity of fungal cell wall.

Conclusion: In this study, the secondary metabolite Azadirachtin and the derivatives are showing inhibitory action against the model protein $1,3 \beta$ glucan synthase and it was suggested that the external application of the ligand and its derivatives can be used because of their poor oral bioavailability.

Keywords: Azadirachtin, 1, $3 \beta$-Glucan synthase, Dermatophytes, Open babel, SWISS ADME, iGEMDOCK, Autodock vina.

(C) 2018 The Authors. Published by Innovare Academic Sciences Pvt Ltd. This is an open access article under the CC BY license (http://creativecommons. org/licenses/by/4. 0/) DOI: http://dx.doi.org/10.22159/ajpcr.2018.v11i1.22171

\section{INTRODUCTION}

Azadirachta indica was commonly known as Neem plant, (synonym melia azadirachta) is an evergreen, fast-growing tree commonly found in arid areas of India, Africa, and America. The neem tree has been described as $A$. indica as early as 1830 by De Jussieu [1], and it belongs to a Family Meliaceae. Every part of the tree has been used in traditional medicine for various human ailments [2-6]. Myriad of secondary metabolites [7] from different parts of the tree have been found to be effective on a wide spectrum of diseases, including dermatophytosis. In the period of Harappa culture around 4500 years back neem was used in medical treatment [8]. A. indica is a small deciduous with a rounded crown with a height of 5-15 $\mathrm{m}$ and a width of 5-7 $\mathrm{m}$ [9]. Due to its more efficacy, better tolerability and null adverse effects, Azadirachtin, a chemical compound belongs to Limonoid group, a tetranortriterpenoid obtained from the neem [10]. Fungal cells are composed of a rigid cell wall, mostly made up of chitin and glucan. $1,3 \beta$-glucan is a major constituent of the fungal cell wall constitutes of about $30-80 \% .1,3$ $\beta$-glucan attached to the core polymer by $1,6 \beta$ branches and forms a branched polymer [11-13]. 1, $3 \beta$-glucan helix is a coiled spring-like structure provides a degree of elasticity and tensile strength to the cell wall [13]. It is the building block for fungal cell wall and is synthesized by $1,3 \beta$-glucan synthase, a well-characterized plasma membraneassociated enzyme with multiple transmembrane domains [11-15]. The enzyme utilizes cytoplasmic UDP-glucose as a substrate and ads up glucose molecules to the growing linear glucan polymer [16]. Whenever required to strengthen the cell wall, the fungi produce 1, $3 \beta$ Glucan by the activation of glucan synthesis. Caspofungin, Micafungin, and Anidulafungin belong to Echinocandin family used in the treatment of various fungal infections [17]. They act by inhibiting 1, $3 \beta$-glucan synthase resulting in cell swelling and cell death of the fungi. The Echinocandins are currently being used for the treatment of life- threatening infections caused by aspergillosis and candidiasis organisms. The novel method of drug discovery is in silico method which helps to identify drug targets with the help of computer-aided bioinformatics software. The software is helpful in analyzing the protein, the target for drug action with possible predicted active site, generate ligands as lead molecule, check for druglikeness, dock the proteins or target with ligand or molecule, hierarchized them based on binding affinities and generating the structure-activity relative (SAR) molecules with physicochemical, druglikeness, and medicinal properties.

\section{METHODS}

\section{Preparation of protein}

$1,3 \beta$ glucan synthase plays a vital role in the synthesis of fungal cell wall. The 3D structure of this protein is not available in PubChem database. The homology modeling of this macromolecule was generated.

\section{Homology modeling}

Homology modeling was developed with the help of software "easy modellar." The query sequence and the template were retrieved from National Center for Biotechnology Information (NCBI), the query sequence was aligned with the template sequence and the model was generated. The generated 3D structure of the macromolecule or model protein was validated by Ramachandran Plot.

\section{Preparation of ligand}

A. indica is known for many secondary metabolites, and they are used in many clinical conditions. From the literature, azadirachtin was selected as the secondary metabolite of $A$. indica. The ligand was directly obtained from PubChem database which is a free database available for compounds for virtual screening. From the PubChem database, the structure was downloaded in .sdf file format. Then .sdf file was converted 
into.pdb file and/or .mol file format with software OPENBABEL.The 105 derivatives (SAR molecules) of the selected secondary metabolite were prepared with the help of SWISS ADME online tool. The same SWISS ADME software was used to generate the physicochemical, pharmacokinetics, medicinal, and druglikeness properties of the secondary metabolite, Azadirachtin and the derivatives. The best suited 10 derivatives were selected from the 105 SAR molecules based on the binding affinity and other chemical properties. Rough docking was performed with iGEMDOCK 2.0 software with a population size of 150 and 70 generations set as default. Lipinski's rule also called as the rule of five (RO5) is a rule of thumb to evaluate the druglikeness or determine if a chemical compound with a certain pharmacological or biological activity has properties that may likely active per orally in human beings.

\section{Components of the rule}

For compounds that have better oral bioavailability, should not violate more than one of the following criteria in Lipinski's rule [18,19]

- No more than five hydrogen bond donors (the total number of nitrogen-hydrogen and oxygen-hydrogen bonds)
- No more than 10 hydrogen bond acceptors (all nitrogen or oxygen atoms)

- A molecular mass $<500$ Daltons

- An octanol-water partition coefficient [20]

- $\log \mathrm{P}$ not $>5$.

\section{Protein-ligand docking}

The protein-ligand docking was performed by autodock vina, an interactive molecular graphics program for calculating and displaying feasible docking modes of pairs of protein and ligands and were presented hierarchically based on binding affinities.

\section{RESULTS}

Protein-ligand preparation

The homology model of target protein, 1, $3 \beta$ glucan synthase was docked with the small molecule called azadirachtin and also with the 105 derivatives or SAR compounds. The homology model of macromolecule or protein or drug target was validated with the Ramachandran plot was shown in Fig. 1. The 10 derivatives were selected based on the

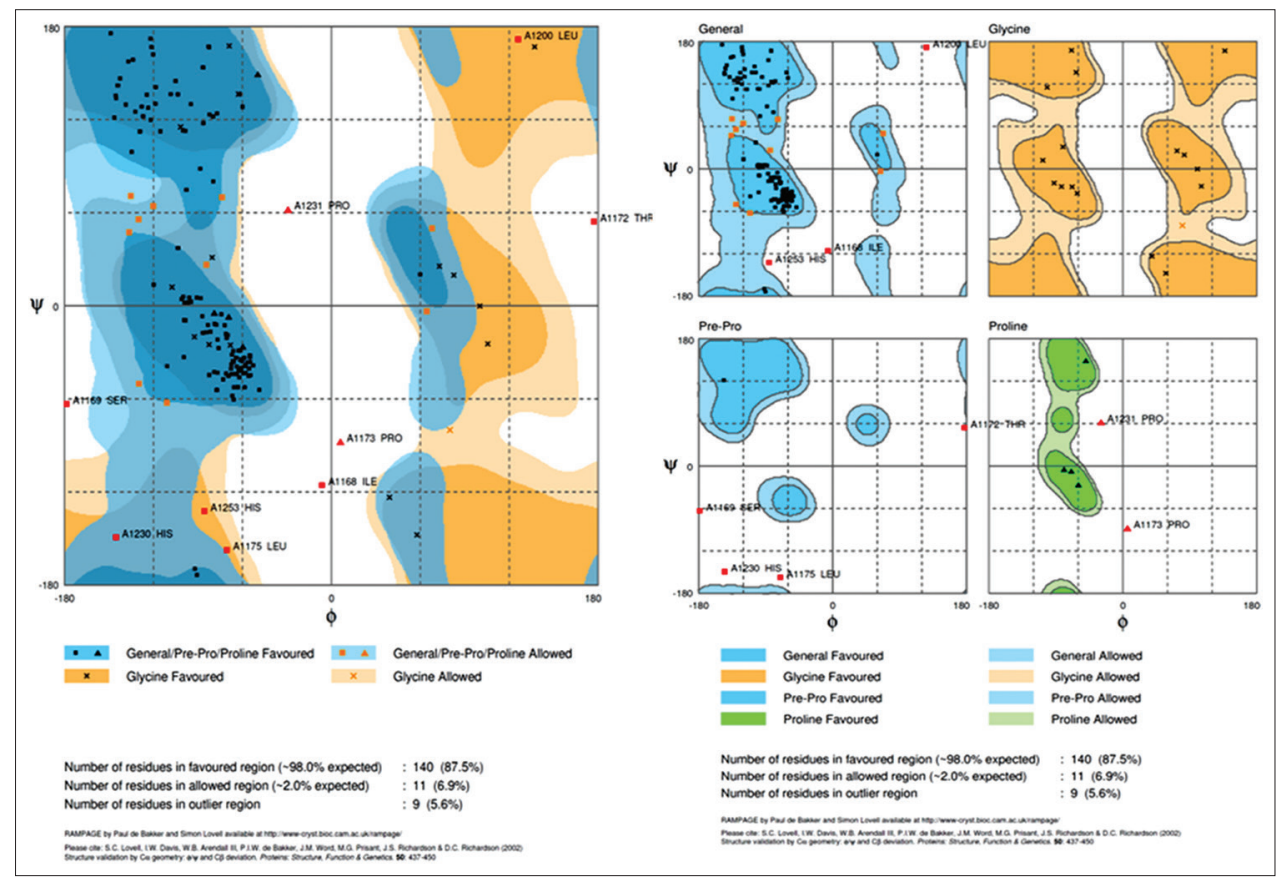

Fig. 1: Ramachandran plot shows the number of amino acids that are favored, allowed and disallowed in model protein 1, $3 \beta$ glucan synthase

Table 1: The results of rough docking was performed with iGEMDOCK in secondary metabolite, azadirachtin and the derivatives

\begin{tabular}{|c|c|c|c|c|c|}
\hline Protein with ligand & $\begin{array}{l}\text { Total energy } \\
\text { (Kcal/mol) }\end{array}$ & $\begin{array}{l}\text { VDW } \\
\text { (Kcal/mol) }\end{array}$ & $\begin{array}{l}\text { H bond } \\
\text { (Kcal/mol) }\end{array}$ & $\begin{array}{l}\text { Electrostatic } \\
\text { (Kcal/mol) }\end{array}$ & $\begin{array}{l}\text { Aver con pair } \\
\text { (Kcal/mol) }\end{array}$ \\
\hline $1,3 \beta$ glucan synthase- azadirachtin & -219.317 & -197.687 & -21.6302 & 0 & 26.5686 \\
\hline 1,3 $\beta$ glucan synthase- azadirachtin D 03 (SAR1) & -152.989 & -139.841 & -13.1479 & 0 & 17.8235 \\
\hline $1,3 \beta$ glucan synthase- azadirachtin D 02 (SAR2) & -239.777 & -225.809 & -13.9683 & 0 & 28.6667 \\
\hline $1,3 \beta$ glucan synthase- azadirachtin D 19 (SAR3) & -173.747 & -170.247 & -3.5 & 0 & 23.9216 \\
\hline $1,3 \beta$ glucan synthase- azadirachtin D 22 (SAR4) & -241 & -232.437 & -8.56344 & 0 & 29.7451 \\
\hline $1,3 \beta$ glucan synthase- azadirachtin D 43 (SAR5) & -186.469 & -184.088 & -2.38063 & 0 & 24.75 \\
\hline 1,3 $\beta$ glucan synthase- azadirachtin D 56 (SAR6) & -143.1 & -119.845 & -23.2549 & 0 & 15.1 \\
\hline $1,3 \beta$ glucan synthase- azadirachtin D 58 (SAR7) & -173.873 & -168.185 & -5.68824 & 0 & 24.6863 \\
\hline $1,3 \beta$ glucan synthase- azadirachtin D 65 (SAR8) & -177.247 & -176.63 & -0.61666 & 0 & 25.56 \\
\hline $1,3 \beta$ glucan synthase- azadirachtin D 70 (SAR9) & -221.85 & -221.85 & 0 & 0 & 27.6275 \\
\hline $1,3 \beta$ glucan synthase- azadirachtin D 81 (SAR10) & -243.297 & -236.12 & -7.17628 & 0 & 29.6923 \\
\hline
\end{tabular}

VDW: Van der Waals force, $\mathrm{H}$ Bond: Hydrogen bond 
binding affinity and the physicochemical, medicinal, and druglikeness properties. The docked poses of the Azadirachtin and the derivatives were shown in Fig. 2. The energy values, Van der Waals force, $\mathrm{H}$-bond were derived by rough docking with iGEMDOCK of the Azadirachtin

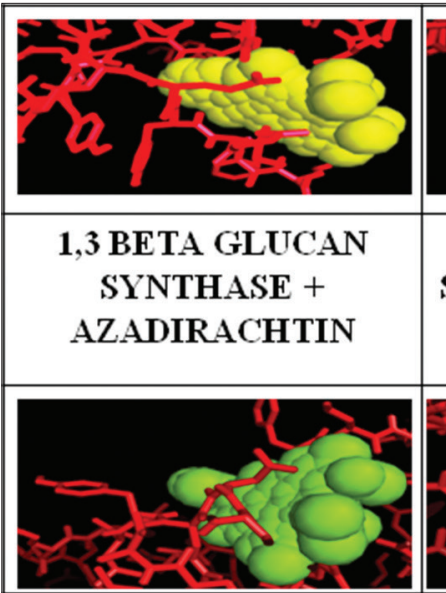

1,3 Beta Glucan Synthase + Azadirachtin Derivative 19 (SAR 3)

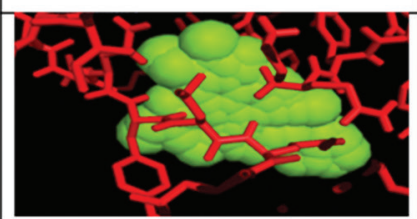

1,3 Beta Glucan Synthase + Azadirachtin Derivative 56 (SAR 6)

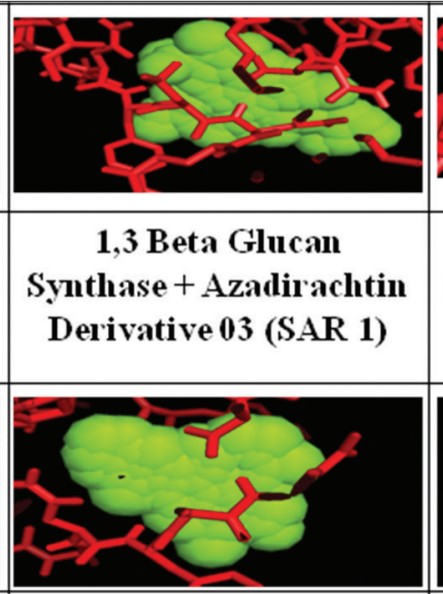

1,3 Beta Glucan Synthase + Azadirachtin Derivative 22 (SAR 4)

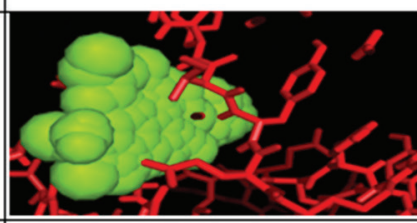

1,3 Beta Glucan Synthase + Azadirachtin Derivative 58 (SAR 7)

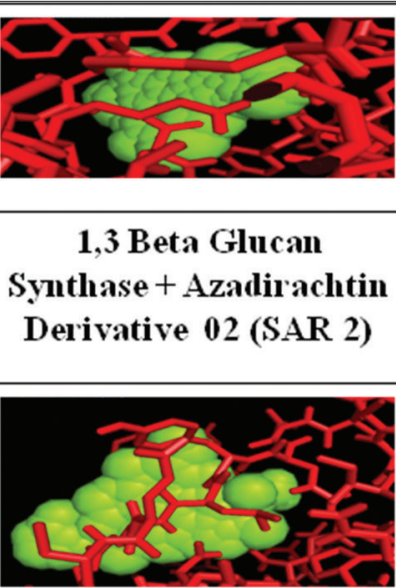

1,3 Beta Glucan Synthase + Azadirachtin Derivative 43 (SAR 5)

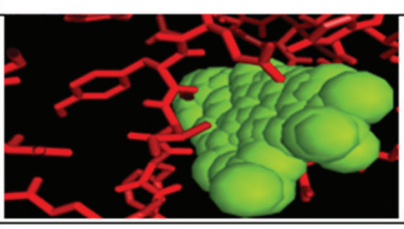

1,3 Beta Glucan Synthase + Azadirachtin Derivative 65 (SAR 8)

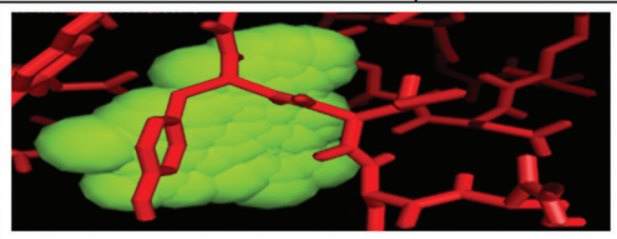

1,3 Beta Glucan Synthase + Azadirachtin Derivative 70 (SAR 9)

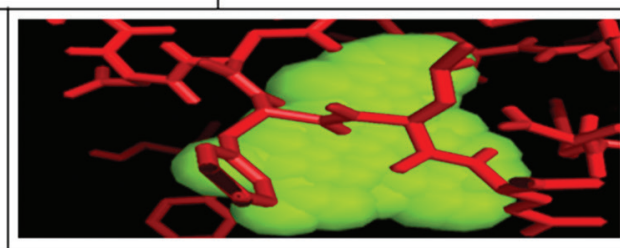

1,3 Beta Glucan Synthase + Azadirachtin Derivative 81 (SAR 10)

Fig. 2: The picture showing the docking poses of secondary metabolite of Azadirachta indica, azadirachtin and the derivatives

Table 2: The results showing the binding affinity of 1, $3 \beta$ Glucan Synthase with Azadirachtin and the derivatives

\begin{tabular}{llc}
\hline Name of the protein and ligand & Binding affinity & RMSD \\
\cline { 2 - 3 } & & Upper bound \\
\hline $1,3 \beta$ glucan synthase-azadirachtin & -13.3 & 0 \\
$1,3 \beta$ glucan synthase -azadirachtin D 03 (SAR1) & -13.9 & 0 \\
$1,3 \beta$ glucan synthase -azadirachtin D 02 (SAR2) & -19.0 & 0 \\
$1,3 \beta$ glucan synthase -azadirachtin D 19 (SAR3) & -13.9 & 0 \\
$1,3 \beta$ glucan synthase -azadirachtin D 22 (SAR4) & -18.4 & 0 \\
$1,3 \beta$ glucan synthase -azadirachtin D 43 (SAR5) & -13.2 & 0 \\
$1,3 \beta$ glucan synthase -azadirachtin D 56 (SAR6) & -14.0 & 0 \\
$1,3 \beta$ glucan synthase -azadirachtin D 58 (SAR7) & -14.9 & 0 \\
$1,3 \beta$ glucan synthase -azadirachtin D 65 (SAR8) & -14.9 & 0 \\
$1,3 \beta$ glucan synthase -azadirachtin D 70 (SAR9) & -18.5 & 0 \\
$1,3 \beta$ glucan synthase -azadirachtin D 81 (SAR10) & -19.1 & 0 \\
\hline
\end{tabular}

RMSD: Root mean square deviation 

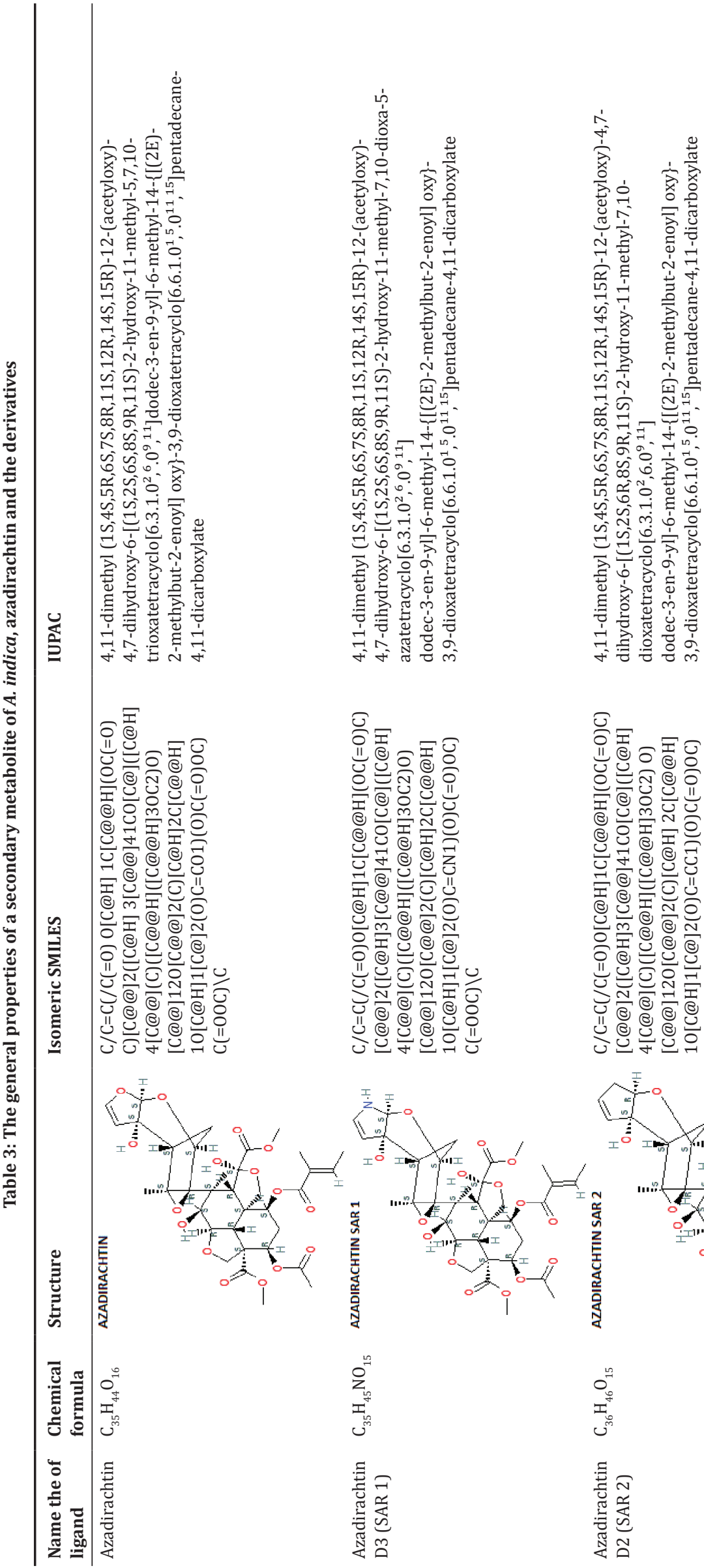

言

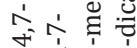

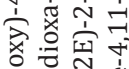

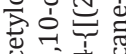

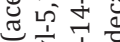

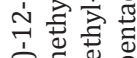

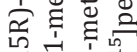

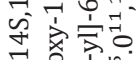
表 ज正的

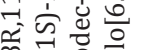
s ते जू. 娄 के ज० जे जें

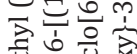
要

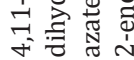
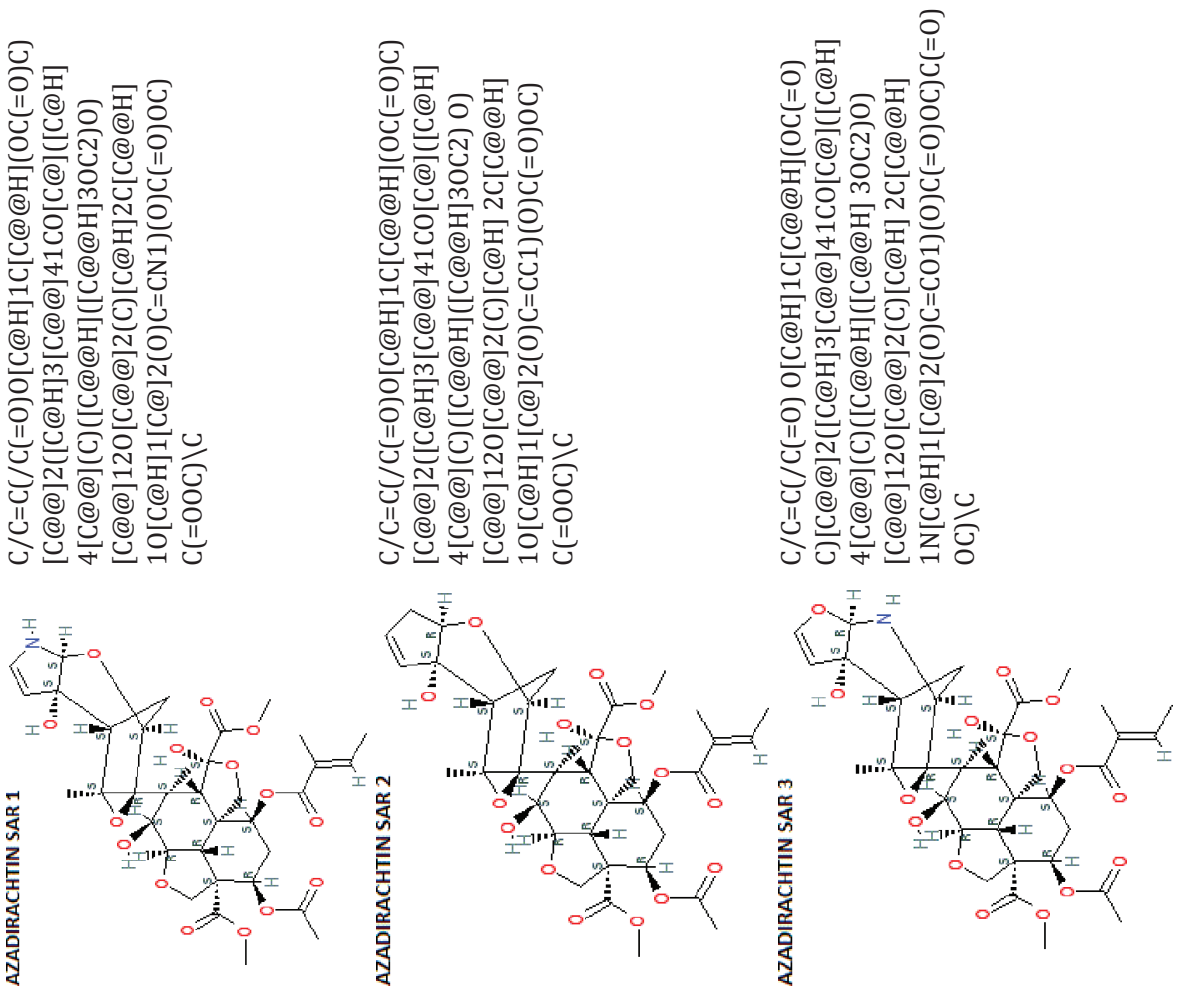

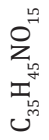

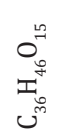

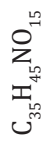

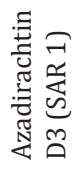

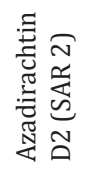

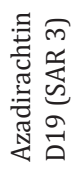




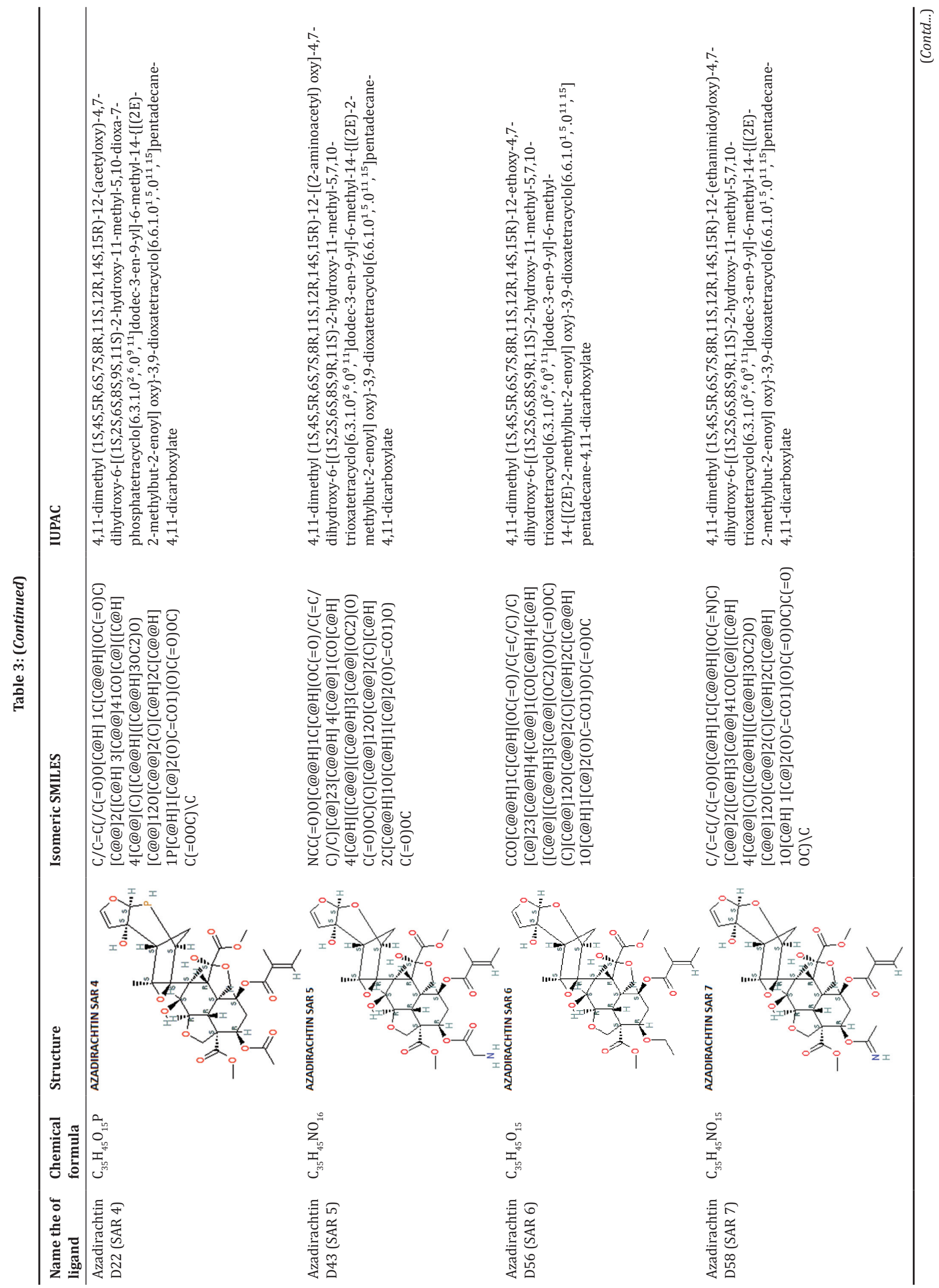




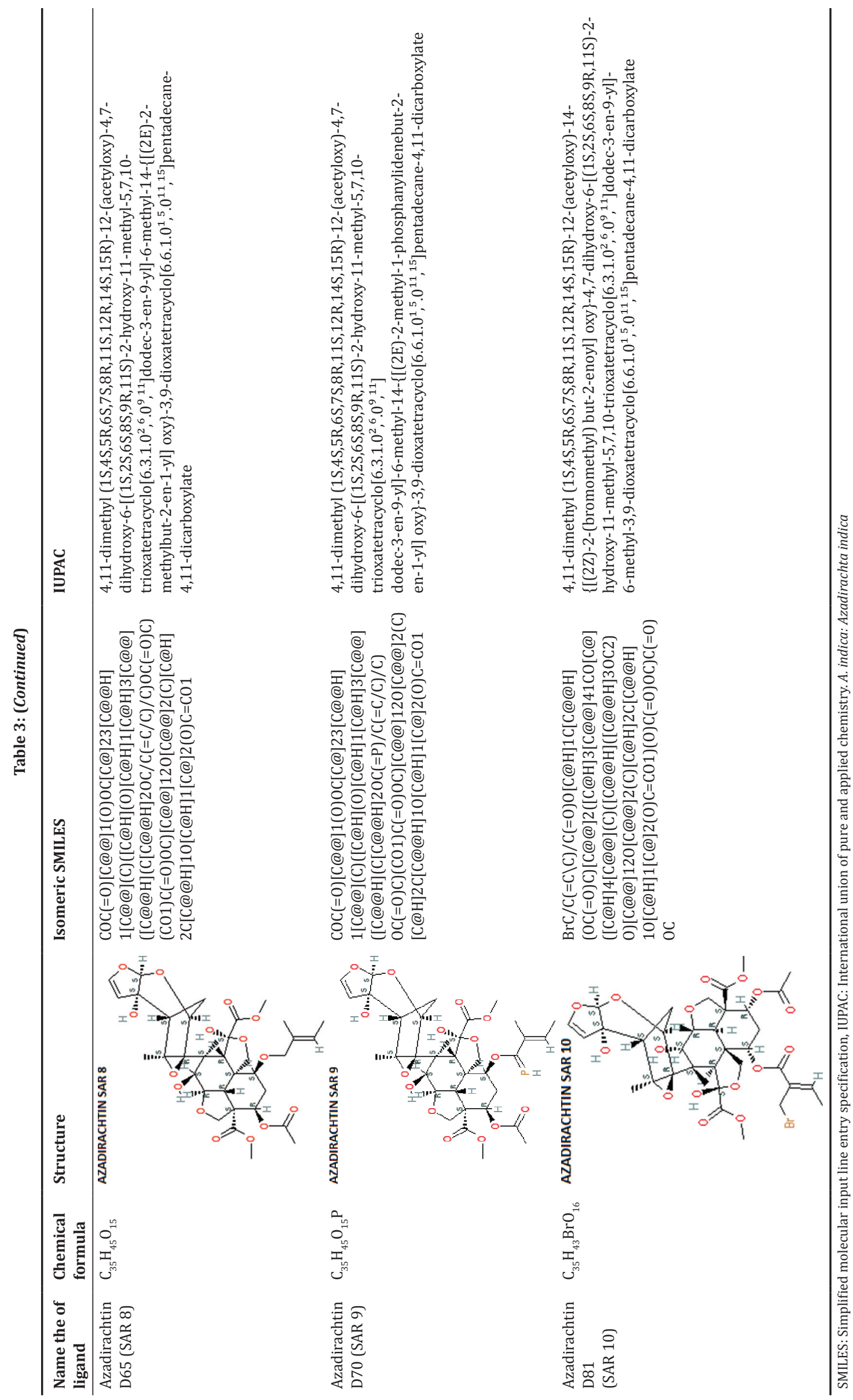


and the derivatives were presented in Table 1 . The binding affinity of the docked protein and ligand was obtained on accurate docking with autodock vina was presented in Table 2. The general properties of azadirachtin and the SAR compounds such as chemical formula, structure, simplified molecular input line entry system (SMILES), and International Union of Pure and Applied Chemistry (IUPAC) were presented in Table 3. The physicochemical properties of the azadirachtin and the SAR compounds such as molecular weight heavy atoms, fraction CSP3, rotatable bonds, H-bond acceptors, H-bond donors, molecular refractivity, and topological polar surface area

Table 4: The physicochemical properties of a secondary metabolite of $A$. indica, azadirachtin and the derivatives

\begin{tabular}{|c|c|c|c|c|c|c|c|c|c|}
\hline Name of the ligand & $\begin{array}{l}\text { Molecular } \\
\text { weight } \\
\text { (g/mol) }\end{array}$ & $\begin{array}{l}\text { Number } \\
\text { heavy } \\
\text { atoms }\end{array}$ & $\begin{array}{l}\text { Number } \\
\text { arom. } \\
\text { heavy } \\
\text { atoms }\end{array}$ & $\begin{array}{l}\text { Fraction } \\
\text { CSP3 }\end{array}$ & $\begin{array}{l}\text { Number } \\
\text { rotatable } \\
\text { bonds }\end{array}$ & $\begin{array}{l}\text { Number } \\
\text { H-bond } \\
\text { acceptors }\end{array}$ & $\begin{array}{l}\text { Number } \\
\text { H-bond } \\
\text { donors }\end{array}$ & $\begin{array}{l}\text { Molar } \\
\text { refractivity }\end{array}$ & TPSA $\left({ }^{\circ} A^{2}\right)$ \\
\hline Azadirachtin & 720.71 & 51 & 0 & 0.77 & 10 & 16 & 3 & 165.92 & 215.34 \\
\hline Azadirachtin D3 (SAR 1) & 719.73 & 51 & 0 & 0.77 & 10 & 15 & 4 & 171.55 & 218.14 \\
\hline Azadirachtin D2 (SAR 2) & 718.74 & 51 & 0 & 0.78 & 10 & 15 & 3 & 169.64 & 206.11 \\
\hline Azadirachtin D19 (SAR 3) & 719.73 & 51 & 0 & 0.77 & 10 & 15 & 4 & 171.55 & 218.14 \\
\hline Azadirachtin D43 (SAR 5) & 735.73 & 52 & 0 & 0.77 & 11 & 17 & 4 & 168.63 & 241.36 \\
\hline Azadirachtin D56 (SAR 6) & 705.72 & 50 & 0 & 0.77 & 10 & 15 & 3 & 166.98 & 198.27 \\
\hline Azadirachtin D58 (SAR 7) & 719.73 & 51 & 0 & 0.77 & 10 & 16 & 4 & 169.31 & 222.12 \\
\hline Azadirachtin D65 (SAR 8) & 705.72 & 50 & 0 & 0.77 & 10 & 15 & 3 & 166.98 & 198.27 \\
\hline Azadirachtin D70 (SAR 9) & 736.7 & 51 & 0 & 0.77 & 10 & 15 & 3 & 174.24 & 232.41 \\
\hline Azadirachtin D81 (SAR 10) & 799.61 & 52 & 0 & 0.77 & 11 & 16 & 3 & 173.79 & 215.34 \\
\hline
\end{tabular}

TPSA: Topological polar surface area, H-bond: Hydrogen bond, A. indica: Azadirachta indica

Table 5: The lipophilicity of a secondary metabolite of $\boldsymbol{A}$. indica, Azadirachtin and the derivatives

\begin{tabular}{|c|c|c|c|c|c|c|}
\hline Name of the ligand & $\begin{array}{l}\text { Log } \\
P_{o / w} \text { (iLOGP) }\end{array}$ & $\begin{array}{l}\text { Log } \\
P_{o / w} \text { (XLOGP) }\end{array}$ & $\begin{array}{l}\text { Log } \\
P_{o / w} \text { (WLOGP) }\end{array}$ & $\begin{array}{l}\text { Log } \\
P_{o / w}(M L O G P)\end{array}$ & $\begin{array}{l}\text { Log } \\
P_{o / w}(\text { SILICOS-IT) }\end{array}$ & $\begin{array}{l}\text { Consensus log } \\
\mathbf{P}_{\text {o/w }} \\
\end{array}$ \\
\hline Azadirachtin & 2.90 & 1.09 & -0.20 & -0.47 & 1.07 & 0.88 \\
\hline Azadirachtin D3 (SAR 1) & 3.51 & 1.1 & -1.01 & -0.47 & 0.76 & 0.78 \\
\hline Azadirachtin D2 (SAR 2) & 4.44 & 1.57 & 0.26 & 0.04 & 1.72 & 1.61 \\
\hline Azadirachtin D19 (SAR 3) & 3.24 & 0.81 & -1.01 & -0.47 & 0.76 & 0.66 \\
\hline Azadirachtin D22 (SAR 4) & 3.67 & 0.93 & 0.46 & -0.14 & 1.1 & 1.2 \\
\hline Azadirachtin D43 (SAR 5) & 4.31 & 0.18 & -1.26 & -1.19 & 0.24 & 0.45 \\
\hline Azadirachtin D58 (SAR 7) & 3.99 & 1.18 & 0.25 & -0.47 & 1.2 & 1.23 \\
\hline Azadirachtin D65 (SAR 8) & 3.53 & 1.36 & 0.06 & -0.55 & 1.51 & 1.18 \\
\hline Azadirachtin D70 (SAR 9) & 3.53 & 0.34 & 0.54 & -0.55 & 0.93 & 0.96 \\
\hline Azadirachtin D81 (SAR 10) & 4.55 & 1.28 & 0.17 & -0.2 & 1.64 & 1.49 \\
\hline
\end{tabular}

o/w: Octanol/water, A. indica: Azadirachta indica

Table 6: The hydrophilicity of a secondary metabolite of $\boldsymbol{A}$. indica, Azadirachtin and the derivatives

\begin{tabular}{|c|c|c|c|c|c|c|c|c|c|}
\hline $\begin{array}{l}\text { Name of the } \\
\text { ligand }\end{array}$ & $\begin{array}{l}\log S \\
(E S O L)\end{array}$ & Solubility & Class & $\begin{array}{l}\log S \\
\text { (Ali) }\end{array}$ & Solubility & Class & $\begin{array}{l}\log S \\
\text { (SILICOS-IT) }\end{array}$ & Solubility & Class \\
\hline Azadirachtin & -4.34 & $\begin{array}{l}3.33 \mathrm{e}-02 \mathrm{mg} / \mathrm{ml} \\
4.62 \mathrm{e}-05 \mathrm{~mol} / \mathrm{l}\end{array}$ & $\begin{array}{l}\text { Moderately } \\
\text { soluble }\end{array}$ & -5.20 & $\begin{array}{l}4.50 \mathrm{e}-03 \mathrm{mg} / \mathrm{ml} \\
6.25 \mathrm{e}-06 \mathrm{~mol} / \mathrm{l}\end{array}$ & $\begin{array}{l}\text { Moderately } \\
\text { soluble }\end{array}$ & -1.40 & $\begin{array}{l}2.86 \mathrm{e}+01 \mathrm{mg} / \mathrm{ml} \\
3.97 \mathrm{e}-02 \mathrm{~mol} / \mathrm{l}\end{array}$ & Soluble \\
\hline $\begin{array}{l}\text { Azadirachtin } \\
\text { D3 (SAR 1) }\end{array}$ & -4.34 & $\begin{array}{l}3.33 \mathrm{E}-02 \mathrm{mg} / \mathrm{ml} \\
4.62 \mathrm{E}-05 \mathrm{~mol} / \mathrm{l}\end{array}$ & $\begin{array}{l}\text { Moderately } \\
\text { soluble }\end{array}$ & -5.27 & $\begin{array}{l}3.83 \mathrm{E}-03 \mathrm{mg} / \mathrm{ml} \\
5.33 \mathrm{E}-06 \mathrm{~mol} / \mathrm{l}\end{array}$ & $\begin{array}{l}\text { Moderately } \\
\text { soluble }\end{array}$ & -1.71 & $\begin{array}{l}1.39 \mathrm{E}+01 \mathrm{mg} / \mathrm{ml} \\
1.94 \mathrm{E}-02 \mathrm{~mol} / \mathrm{l}\end{array}$ & Soluble \\
\hline $\begin{array}{l}\text { Azadirachtin } \\
\text { D2 (SAR 2) }\end{array}$ & -4.63 & $\begin{array}{l}1.70 \mathrm{E}-02 \mathrm{mg} / \mathrm{ml} \\
2.37 \mathrm{E}-05 \mathrm{~mol} / \mathrm{l}\end{array}$ & $\begin{array}{l}\text { Moderately } \\
\text { soluble }\end{array}$ & -5.51 & $\begin{array}{l}2.23 \mathrm{E}-03 \mathrm{mg} / \mathrm{ml} \\
3.10 \mathrm{E}-06 \mathrm{~mol} / \mathrm{l}\end{array}$ & $\begin{array}{l}\text { Moderately } \\
\text { soluble }\end{array}$ & -1.94 & $\begin{array}{l}8.21 \mathrm{E}+00 \mathrm{mg} / \mathrm{ml} \\
1.14 \mathrm{E}-02 \mathrm{~mol} / \mathrm{l}\end{array}$ & Soluble \\
\hline $\begin{array}{l}\text { Azadirachtin } \\
\text { D22 (SAR 4) }\end{array}$ & -4.33 & $\begin{array}{l}3.42 \mathrm{E}-02 \mathrm{mg} / \mathrm{ml} \\
4.64 \mathrm{E}-05 \mathrm{~mol} / \mathrm{l}\end{array}$ & $\begin{array}{l}\text { Moderately } \\
\text { soluble }\end{array}$ & -5.13 & $\begin{array}{l}5.46 \mathrm{E}-03 \mathrm{mg} / \mathrm{ml} \\
7.41 \mathrm{E}-06 \mathrm{~mol} / \mathrm{l}\end{array}$ & $\begin{array}{l}\text { Moderately } \\
\text { soluble }\end{array}$ & -1.75 & $\begin{array}{l}1.32 \mathrm{E}+01 \mathrm{mg} / \mathrm{ml} \\
1.79 \mathrm{E}-02 \mathrm{~mol} / \mathrm{l}\end{array}$ & Soluble \\
\hline $\begin{array}{l}\text { Azadirachtin } \\
\text { D43 (SAR 5) }\end{array}$ & -3.79 & $\begin{array}{l}1.20 \mathrm{E}-01 \mathrm{mg} / \mathrm{ml} \\
1.63 \mathrm{E}-04 \mathrm{~mol} / \mathrm{l}\end{array}$ & Soluble & -4.81 & $\begin{array}{l}1.15 \mathrm{E}-02 \mathrm{mg} / \mathrm{ml} \\
1.56 \mathrm{E}-05 \mathrm{~mol} / \mathrm{l}\end{array}$ & $\begin{array}{l}\text { Moderately } \\
\text { soluble }\end{array}$ & -1.04 & $\begin{array}{l}6.77 \mathrm{E}+01 \mathrm{mg} / \mathrm{ml} \\
9.20 \mathrm{E}-02 \mathrm{~mol} / \mathrm{l}\end{array}$ & Soluble \\
\hline $\begin{array}{l}\text { Azadirachtin } \\
\text { D56 (SAR 6) }\end{array}$ & -4.45 & $\begin{array}{l}2.50 \mathrm{E}-02 \mathrm{mg} / \mathrm{ml} \\
3.55 \mathrm{E}-05 \mathrm{~mol} / \mathrm{l}\end{array}$ & $\begin{array}{l}\text { Moderately } \\
\text { soluble }\end{array}$ & -5.19 & $\begin{array}{l}4.57 \mathrm{E}-03 \mathrm{mg} / \mathrm{ml} \\
6.48 \mathrm{E}-06 \mathrm{~mol} / \mathrm{l}\end{array}$ & $\begin{array}{l}\text { Moderately } \\
\text { soluble }\end{array}$ & -1.88 & $\begin{array}{l}9.35 \mathrm{E}+00 \mathrm{mg} / \mathrm{ml} \\
1.32 \mathrm{E}-02 \mathrm{~mol} / \mathrm{l}\end{array}$ & Soluble \\
\hline $\begin{array}{l}\text { Azadirachtin } \\
\text { D58 (SAR 7) }\end{array}$ & -4.39 & $\begin{array}{l}2.96 \mathrm{E}-02 \mathrm{mg} / \mathrm{ml} \\
4.11 \mathrm{E}-05 \mathrm{~mol} / \mathrm{l}\end{array}$ & $\begin{array}{l}\text { Moderately } \\
\text { soluble }\end{array}$ & -5.44 & $\begin{array}{l}2.61 \mathrm{E}-03 \mathrm{mg} / \mathrm{ml} \\
3.63 \mathrm{E}-06 \mathrm{~mol} / \mathrm{l}\end{array}$ & $\begin{array}{l}\text { Moderately } \\
\text { soluble }\end{array}$ & -1.55 & $\begin{array}{l}2.04 \mathrm{E}+01 \mathrm{mg} / \mathrm{ml} ; \\
2.84 \mathrm{E}-02 \mathrm{~mol} / \mathrm{l}\end{array}$ & Soluble \\
\hline $\begin{array}{l}\text { Azadirachtin } \\
\text { D65 (SAR 8) }\end{array}$ & -4.41 & $\begin{array}{l}2.73 \mathrm{E}-02 \mathrm{mg} / \mathrm{ml} \\
3.87 \mathrm{E}-05 \mathrm{~mol} / \mathrm{l}\end{array}$ & $\begin{array}{l}\text { Moderately } \\
\text { soluble }\end{array}$ & -5.13 & $\begin{array}{l}5.28 \mathrm{E}-03 \mathrm{mg} / \mathrm{ml} \\
7.48 \mathrm{E}-06 \mathrm{~mol} / \mathrm{l}\end{array}$ & $\begin{array}{l}\text { Moderately } \\
\text { soluble }\end{array}$ & -1.88 & $\begin{array}{l}9.35 \mathrm{E}+00 \mathrm{mg} / \mathrm{ml} \\
1.32 \mathrm{E}-02 \mathrm{~mol} / \mathrm{l}\end{array}$ & Soluble \\
\hline $\begin{array}{l}\text { Azadirachtin } \\
\text { D70 (SAR 9) }\end{array}$ & -3.96 & $\begin{array}{l}8.05 \mathrm{E}-02 \mathrm{mg} / \mathrm{ml} \\
1.09 \mathrm{E}-04 \mathrm{~mol} / \mathrm{l}\end{array}$ & Soluble & -4.78 & $\begin{array}{l}1.21 \mathrm{E}-02 \mathrm{mg} / \mathrm{ml} \\
1.64 \mathrm{E}-05 \mathrm{~mol} / \mathrm{l}\end{array}$ & $\begin{array}{l}\text { Moderately } \\
\text { soluble }\end{array}$ & -1.58 & $\begin{array}{l}1.94 \mathrm{E}+01 \mathrm{mg} / \mathrm{ml} ; \\
2.64 \mathrm{E}-02 \mathrm{~mol} / \mathrm{l}\end{array}$ & Soluble \\
\hline $\begin{array}{l}\text { Azadirachtin } \\
\text { D81 (SAR 10) }\end{array}$ & -4.88 & $\begin{array}{l}1.06 \mathrm{E}-02 \mathrm{mg} / \mathrm{ml} \\
1.32 \mathrm{E}-05 \mathrm{~mol} / \mathrm{l}\end{array}$ & $\begin{array}{l}\text { Moderately } \\
\text { soluble }\end{array}$ & -5.4 & $\begin{array}{l}3.17 \mathrm{E}-03 \mathrm{mg} / \mathrm{ml} \\
3.97 \mathrm{E}-06 \mathrm{~mol} / \mathrm{l}\end{array}$ & $\begin{array}{l}\text { Moderately } \\
\text { soluble }\end{array}$ & -2.15 & $\begin{array}{l}5.71 \mathrm{E}+00 \mathrm{mg} / \mathrm{ml} \\
7.14 \mathrm{E}-03 \mathrm{~mol} / \mathrm{l}\end{array}$ & Soluble \\
\hline
\end{tabular}

A. indica: Azadirachta indica 
Table 7: The pharmacokinetics properties of a secondary metabolite of $A$. indica, azadirachtin and the derivatives

\begin{tabular}{|c|c|c|c|c|c|c|c|c|c|}
\hline Name of ligand & $\begin{array}{l}\text { GI } \\
\text { absorption }\end{array}$ & $\begin{array}{l}\text { BBB } \\
\text { permeability }\end{array}$ & $\begin{array}{l}\text { P-gp } \\
\text { substrate }\end{array}$ & $\begin{array}{l}\text { CYP 1A2 } \\
\text { inhibitor }\end{array}$ & $\begin{array}{l}\text { CYP 2C19 } \\
\text { inhibitor }\end{array}$ & $\begin{array}{l}\text { CYP 2C9 } \\
\text { inhibitor }\end{array}$ & $\begin{array}{l}\text { CYP 2D6 } \\
\text { inhibitor }\end{array}$ & $\begin{array}{l}\text { CYP 3A4 } \\
\text { inhibitor }\end{array}$ & $\begin{array}{l}\log K_{p} \text { (skin } \\
\text { permeation) } \mathrm{cm} / \mathrm{s}\end{array}$ \\
\hline Azadirachtin & Low & No & Yes & No & No & No & No & No & -9.92 \\
\hline Azadirachtin D3 (SAR 1) & Low & No & Yes & No & No & No & No & No & -9.91 \\
\hline Azadirachtin D2 (SAR 2) & Low & No & Yes & No & No & No & No & No & -9.57 \\
\hline Azadirachtin D19 (SAR 3) & Low & No & Yes & No & No & No & No & No & -10.12 \\
\hline Azadirachtin D22 (SAR 4) & Low & No & Yes & No & No & No & No & No & -10.13 \\
\hline Azadirachtin D43 (SAR 5) & Low & No & Yes & No & No & No & No & No & -10.66 \\
\hline Azadirachtin D58 (SAR 7) & Low & No & Yes & No & No & No & No & No & -9.85 \\
\hline Azadirachtin D65 (SAR 8) & Low & No & Yes & No & No & No & No & No & -9.64 \\
\hline Azadirachtin D70 (SAR 9) & Low & No & Yes & No & No & No & No & No & -10.55 \\
\hline Azadirachtin D81 (SAR 10) & Low & No & Yes & No & No & No & No & No & -10.27 \\
\hline
\end{tabular}

GI absorption: Gastrointestinal absorption, BBB: Blood brain barrier, CYP: Cytochrome P, A. indica: Azadirachta indica

Table 8: The druglikeness of a secondary metabolite of $\boldsymbol{A}$. indica, azadirachtin and the derivatives

\begin{tabular}{|c|c|c|c|c|c|c|}
\hline Name of the ligand & Lipinski & Ghose & Veber & Egan & Muegge & Bioavailability score \\
\hline Azadirachtin & 2 violations & 3 violations & 1 violation & 1 violation & 4 violations & 0.17 \\
\hline Azadirachtin D3 (SAR 1) & 2 violations & 4 violations & 1 violations & 1 violations & 4 violations & 0.17 \\
\hline Azadirachtin D2 (SAR 2) & 2 violations & 3 violations & 1 violations & 1 violations & 4 violations & 0.17 \\
\hline Azadirachtin D19 (SAR 3) & 2 violations & 4 violations & 1 violations & 1 violations & 4 violations & 0.17 \\
\hline Azadirachtin D22 (SAR 4) & 2 violations & 3 violations & 1 violations & 1 violations & 4 violations & 0.17 \\
\hline Azadirachtin D43 (SAR 5) & 2 violations & 4 violations & 2 violations & 1 violations & 4 violations & 0.17 \\
\hline Azadirachtin D56 (SAR 6) & 2 violations & 3 violations & 1 violations & 1 violations & 4 violations & 0.17 \\
\hline Azadirachtin D65 (SAR 8) & 2 violations & 3 violations & 1 violations & 1 violations & 4 violations & 0.17 \\
\hline Azadirachtin D70 (SAR 9) & 2 violations & 3 violations & 1 violations & 1 violations & 4 violations & 0.17 \\
\hline Azadirachtin D81 (SAR 10) & 2 violations & 3 violations & 2 violations & 1 violations & 4 violations & 0.17 \\
\hline
\end{tabular}

A. indica: Azadirachta indica

Table 9: The toxicity of a secondary metabolite of $A$. indica, azadirachtin and the derivatives

\begin{tabular}{|c|c|c|c|c|c|}
\hline Name of the ligand & hERG inhibition & AMES toxicity & Carcinogens & Acute oral toxicity & Rat acute toxicity (LD $50 \mathrm{mg} /$ ) \\
\hline Azadirachtin & 0.9919 & 0.7563 & 0.9455 & 0.6952 & 4.3477 \\
\hline Azadirachtin D3 (SAR 1) & 0.9969 & 0.5690 & 0.9550 & 0.4926 & 3.0765 \\
\hline Azadirachtin D2 (SAR 2) & 0.9919 & 0.7563 & 0.9455 & 0.6952 & 4.3477 \\
\hline Azadirachtin D19 (SAR 3) & 0.9972 & 0.5171 & 0.9455 & 0.4294 & 3.1422 \\
\hline Azadirachtin D22 (SAR 4) & 0.9917 & 0.7483 & 0.9503 & 0.5852 & 3.8622 \\
\hline Azadirachtin D43 (SAR 5) & 0.9805 & 0.6369 & 0.9330 & 0.3630 & 3.6150 \\
\hline Azadirachtin D56 (SAR 6) & 0.9887 & 0.6849 & 0.9393 & 0.6161 & 4.2870 \\
\hline Azadirachtin D65 (SAR 8) & 0.9880 & 0.7287 & 0.9470 & 0.7958 & 4.7577 \\
\hline Azadirachtin D70 (SAR 9) & 0.9908 & 0.7386 & 0.9449 & 0.6280 & 4.1677 \\
\hline Azadirachtin D81 (SAR 10) & 0.9907 & 0.6846 & 0.9346 & 0.6098 & 4.2446 \\
\hline
\end{tabular}

hERG: Human ether-a-go-go-related gene, $A$. indica: Azadirachta indica

(TPSA) were presented in Table 4. The lipophilicity and hydrophilicity of azadirachtin and the SAR compounds were shown in Tables 5 and 6, respectively. The pharmacokinetic properties of azadirachtin and the SAR compounds were presented in Table 7. The druglikeness of the azadirachtin and the SAR compounds were shown in Table 8.

\section{Ramachandran plot}

The Ramachandran plot is the way to visualize the dihedral angles $\psi$ (phi) and $\varphi$ (psi) of a protein backbone [21] was discovered by Ramachandran et al. [22]. Due to steric hindrances that occur between adjacent atoms within a protein structure, the $\psi$ (phi) and $\varphi$ (psi) values are usually constrained within specific areas of the plot, particularly for ordered structures such as helices and sheets. The dihedral angles or torsion angles for loop regions in a given protein do not often occupy particular regions in the plot unlike secondary structure elements such as $\alpha$-helices or $\beta$-sheets, but they may occupy any regions that are sterically permitted. The $1,3 \beta$ glucan synthase protein structure was validated using procheck and from the Ramachandran plot, it was inferred that the modeled protein contains $87.5 \%$ of amino acid residues in the favored region, $6.9 \%$ in allowed region, and 5.6\% in amino acid residues in disallowed region.
In Table 1, the secondary metabolite, azadirachtin shows energy values as -219.317 and Van der Waals force -197.687 between protein and ligand. The SAR 10 was showing more than the secondary metabolite as energy values -243.297 and Van der Waals force -236.12 .

In Table 2 summarizes that the binding affinity between protein and ligand for azadirachtin was -13.3 and the SAR 10 molecule was -19.1. The more energy value, Van der Waals force, and binding affinity between protein and ligand show more likely to be a new drug entity.

Table 3 summarizes the general properties such as molecular formula, chemical structure, simplified molecular input line entry specification (SMILES), and IUPAC name of a secondary metabolite of $A$. indica, azadirachtin and the derivatives.

In Table 4 showing molecular weight, number of atoms, fraction CSP3, number of rotatable bonds, molar refractivity, and TPSA, where it shows that the molecular weight is more than 500 , number of atoms are in the permissible range of $20-70$, molar refractivity is more than 130 , polar 
surface area is also more than 140 angstroms squared in Azadirachtin and also the derivatives implies that it is a poor oral bioavailability.

Table 5 is showing the $\log \mathrm{p}$ octanol-water partition coefficient values of the azadirachtin, and the derivatives are in the range of permissible $-0.4-+5.6$ range that implies a good lipophilic compounds. The consensus $\log \mathrm{p}_{\mathrm{o} / \mathrm{w}}$ means an average of all five predictions is also in the permissible range.

The Table 6 is showing the hydrophilicity property of the azadirachtin and the derivatives which are mostly moderately soluble.

Table 7 is showing the pharmacokinetic property of azadirachtin, and the derivatives implies the oral bioavailability is poor, and drug penetration to skin is high.

From the Table 8 summarizes that the azadirachtin and the derivatives do not obey the Lipinski's rule of 5 and other filters for a new drug molecule, and the bioavailability score is also very low. This implies that the oral bioavailability of these compounds is poor.

Table 9 summarizes the toxicity of azadirachtin and the derivatives, which these compounds are non-toxic in hERG, AMES, carcinogenicity, acute oral toxicity, and LD50 in rats.

\section{DISCUSSION}

Fungi are ubiquitous constitute a very diverse group of organisms. They evolved and adapted to live in a wide variety of environmental and ecological niches. Most of the fungal infections in human beings are superficial and relatively innocuous, but some can cause devastating diseases such as invasive aspergillosis and systemic candidiasis. The currently available drugs for fungal diseases are amphotericin B, nystatin, griseofulvin, flucytosine, clotrimazole, miconazole, ketoconazole, fluconazole, terbinafine, tolnaftate, salicylic acid, and benzoic acid [23]. Medicinal plants are very widely used in modern days as these are safe and devoid of untoward events. A. indica is one of the plants having a myriad of medicinal properties. The whole plant is used against human ailments, especially for infections caused by bacteria, fungi, and viral organisms. New drug development is a tedious process which takes $15-20$ years to develop a successful new drug entity. In silico method of drug discovery is helping us to discover newer ligands or molecules or drug substances which can reduce the pre-clinical study period. $1,3 \beta$ glucan synthase, a model protein selected from literature as a drug target whose 3D structure was not available in NCBI. The homology was generated with easy modellar and it was validated with Procheck of Ramachandran plot. Azadirachtin, the secondary metabolite was selected as ligand. The protein and ligand were docked with iGEMDOCK and Autodock Vina [24], the results were retrieved on the basis of energy values, Van der Waals force, binding affinity between protein and ligand. The SAR molecules were generated with the help of SWISS ADME [25-27] online tool. In a study by Jeyam et al. showed that a good interaction between 1,3 $\beta$ glucan synthase with 20 phytoconstituents and the inhibition of $1,3 \beta$ glucan synthase was better than echinocandins [28]. According to Juan, homoallylamines displays similar and stronger antifungal activity by inhibiting $1,3 \beta$ glucan synthase against Epidermophyton floccosum and Microsporum canis with amphotericin B and ketoconazole [29]. Onishi stated that lipopeptide antifungal agents are potential therapeutic agents against aspergillosis and candidiasis by inhibiting 1,3 $\beta$ glucan synthase [30]. In this study also it was observed a good interaction between $1,3 \beta$ glucan synthase with azadirachtin and the derivatives.

\section{CONCLUSION}

In this study, the secondary metabolite azadirachtin and the derivatives are showing inhibitory action against the model protein $1,3 \beta$ glucan synthase. It was suggested that the protein-ligand interaction for a new drug entity between the $1,3 \beta$ glucan synthase and azadirachtin or with
SARs were more reliable as external application being they are having poor oral bioavailability.

\section{ACKNOWLEDGMENT}

The authors are thankful for the management of Tagore Medical College and Hospital, Rathinamangalam, Chennai, and Central Research Lab, Tagore Medical College and Hospital, for providing the required software.

\section{CONFLICT OF INTEREST}

Declarared none

\section{AUTHORS CONTRIBUTION}

Simhadri.V.S.D.N.A.Nagesh - Principle Investigator

DR. M. Muniappan- Guide

DR. I. Kannan - Co-Guide

DR. S. Viswanathan - Provided Required Software for Study

\section{REFERENCES}

1. de Jussieu A. Mémoire sur le groupe des Méliacéas. Mem Mus Hist Nat Paris 1830;19:153-304

2. Rao AD, Devi KN, Thyagaraju K. Mechanism of antioxidant activity in neem. J Enzyme Inhib 1998;14:85-6.

3. Tidjani MA, Dupont C, Wepierre J. Azadirachta indica stem bark extract anti-inflammatory activity. Planta Med Phytother 1989;23:259-66.

4. Khan M, Wassilew SW, Schmutterer H, Ascher KR. Natural Pesticides From the Neem Tree and Other Tropical Plants. Eschborn, Germany: Proceedings of $3^{\text {rd }}$ International Neem Conference; 1987. p. 460-6.

5. Maragathavalli S, Brindha S, Kaviyarasi NS, Annadurai B, Gangwar SK. Antimicrobial activity in leaf extract of Neem (Azadirachta indica Linn). Int J Sci Nat 2012;3:110-3

6. Almas K. The antimicrobial effects of extracts of Azadirachta indica (Neem) and Salvadora persica (Arak) chewing sticks. Indian J Dent Res 1999;10:23-6.

7. Subapriya R, Nagini S. Medicinal properties of neem leaves: A review. Curr Med Chem Anticancer Agents 2005;5:149-6.

8. Krishnan Y, Wong NK. Cytotoxicity and antimicrobial properties of neem (Azadirachta indica) leaf extracts. Int J Pharm Pharm Sci 2015;7:179-82.

9. Jaafar NS, Hamad MN, Abbas IS, Jaafar IS. Qualitative phytochemical comparison between flavonoids and phenolic acids contents of leaves and fruits of Melia azedarach (Family: Meliaceae) cultivated in Iraq by HPLC and HPTLC. Int J Pharm Pharm Sci 2016;8:242-50.

10. Locke JC. Fungi. In: Schmutterer H, editor. The Neem Tree. Weinheim, Germany: VHC; 1995. p. 118-26.

11. Klis FM, Boorsma A, de Groot PW. Cell wall construction in Saccharomyces cerevisiae. Yeast 2006;23:185-202.

12. Latgé JP. The cell wall: A carbohydrate armour for the fungal cell. Mol Microbiol 2007;66:279-90.

13. Lesage G, Bussey H. Cell wall assembly in Saccharomyces cerevisiae. Microbiol Mol Biol Rev 2006;70:317-43.

14. Douglas CM, Foor F, Marriman JA, Morin N, Nielsen JB, Dahl AM, et al. The Saccharomyces cerevisiae FKS1 (ETG1) gene encodes an integral membrane protein which is a subunit of 1,3-b-D-glucan synthase. Proc Natl Acad Sci USA 1994:91:12907-11.

15. Thompson JR, Douglas CM, Li W, Jue CK, Pramanik B, Yuan X, et al. A glucan synthase FKS1 homolog in cryptococcus neoformans is single copy and encodes an essential function. J Bacteriol 1999;181:444-53.

16. Frost DJ, Brandt K, Capobianco J, Goldman R. Characterization of (1,3)-beta-glucan synthase in Candida albicans: Microsomal assay from the yeast or mycelial morphological forms and a permeabilized whole-cell assay. Microbiology 1994;140:2239-46.

17. Chen SC, Slavin MA, Sorrell TC. Echinocandin antifungal drugs in fungal infections. Drugs 2011;71:11-41.

18. Lipinski CA, Lombardo F, Dominy BW, Feeney PJ. Experimental and computational approaches to estimate solubility and permeability in drug discovery and development settings. Adv Drug Deliv Rev 2001:46:3-26.

19. Lipinski CA. Lead-and drug-like compounds: The rule-of-five revolution. Drug Discov Today Technol 2004;1:337-41.

20. Leo A, Hansch C, Elkins D. Partition coefficients and their uses. Chem 
Rev 1971;71:525-616

21. Richardson JS. The anatomy and taxonomy of protein structure. Adv Protein Chem 1981;34:167-339.

22. Ramachandran GN, Ramakrishnan C, Sasisekharan V. Stereochemistry of polypeptide chain configurations. J Mol Biol 1963;7:95-9.

23. Baron S, Dixon DM, Walsh TJ. Antifungal agents. Medical Microbiology. $4^{\text {th }}$ ed. Ch. 76. Galveston: University of Texas Medical Branch; 1996.

24. Trott O, Olson AJ. AutoDock Vina: Improving the speed and accuracy of docking with a new scoring function, efficient optimization, and multithreading. J Comput Chem 2010;31:455-61.

25. Daina A, Michielin O, Zoete V. SwissADME: A free web tool to evaluate pharmacokinetics, druglikeness and medicinal chemistry friendliness of small molecules. Sci Rep 2017;7:42717.

26. Daina A, Michielin O, Zoete V. iLOGP: A simple, robust, and efficient description of n-octanol/water partition coefficient for drug design using the GB/SA approach. J Chem Inf Model 2014;54:3284-301.

27. Daina A, Zoete V. A BOILED-egg to predict gastrointestinal absorption and brain penetration of small molecules. ChemMedChem 2016;11:1117-21.

28. Jeyam M, Arangaraj M, Ravikumar P, Shalini G. Computational analysis of phytocompounds with $1,3-\beta-D-G l u c a n$ synthase for antidermatophytic activity. J Appl Pharm Sci 2014;4:64-9.

29. Urbina JM, Cortés JC, Palma A, López SN, Zacchino SA, Enriz RD, et al. Inhibitors of the fungal cell wall. Synthesis of 4-Aryl-4-Narylamine-1-butenes and related compounds with inhibitory activities on $\beta(1-3)$ glucan and chitin synthases. Bioorg Med Chem 2000;8:691-8.

30. Onishi J, Meinz M, Thompson J, Curotto J, Dreikorn S, Rosenbach M, et al. Discovery of novel antifungal $(1,3)$-beta-D-glucan synthase inhibitors. Antimicrob Agents Chemother 2000;44:368-77. 